\title{
Dependence of nonlinear elasticity on filler size in composite polymer systems
}

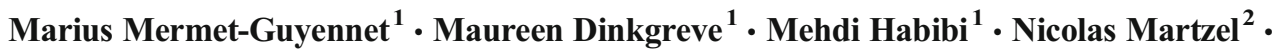 \\ Rudolf Sprik $^{1} \cdot$ Morton Denn $^{3} \cdot$ Daniel Bonn $^{1}$
}

Received: 18 October 2016/Revised: 1 April 2017 / Accepted: 1 April 2017/Published online: 5 May 2017

(C) The Author(s) 2017. This article is an open access publication

\begin{abstract}
Nanosized filler particles enhance the mechanical properties of polymer composites in a size-dependent fashion. This is puzzling, because classical elasticity is inherently scale-free, and models for the elasticity of composite systems never predict a filler-size dependence. Here, we study the industrially important system of silica-filled rubbers, together with a well-characterized model-filled crosslinked gel and show that at high filler content both the linear and nonlinear elastic properties of these systems exhibit a unique scaling proportional to the cube of the volume fraction divided by the particle size. This remarkable behavior makes it possible to predict the full mechanical response of particle-filled rubbers for small but finite deformations based solely on the rheology of the matrix and the size and modulus of the filler particles.
\end{abstract}

Keywords Normal stress $\cdot$ Reinforcement $\cdot$ Filled rubbers · Composites $\cdot$ Nanoparticles

\section{Introduction}

Adding particulate fillers to a polymer matrix dramatically changes the mechanical behavior (Fröhlich, et al. 2005,

Marius Mermet-Guyennet

m.r.b.mermet-guyennet@uva.nl

1 Institute of Physics, University of Amsterdam, Science Park 904, 1098XH Amsterdam, The Netherlands

2 Manufacture Française des Pneumatiques MICHELIN, Site de Ladoux, 23 place Carmes Déchaux, 63040 Clermont Ferrand, France

3 Benjamin Levich Institute and Department of Chemical Engineering, The City College of New York, CUNY, New York, NY 10031, USA
Mermet-Guyennet, et al. 2015, Mermet-Guyennet, et al. 2016, Payne 1962, Rattanasom, et al. 2007). The physical properties of synthetic rubbers that are important for tire performance, for example, depend on the addition of fillers, typically silica or carbon black. It is known that composite properties may improve substantially at a constant volume fraction of filler as the filler size is decreased, yet classical elasticity is inherently scale-free, and continuum models for the elasticity of composite systems never predict a filler-size dependence (Chen, et al. 2015, Guth 1945, Raos 2003, Sadd 2009, Smallwood 1944, Treloar 1975). We show here a remarkable scaling incorporating particle size and volume fraction that applies to the rheological properties of two rubbers with silica fillers, as well as to a filled crosslinked gel that serves as a good model material.

We have recently (Mermet-Guyennet et al. 2016) shown that the linear viscoelastic reinforcement for two filled rubbers and the model crosslinked gel, defined as the ratio of the storage modulus of the filled material to that of the matrix less 1 and denoted $R_{\mathrm{LVE}}$, can be fit by the following equation:

$$
R_{\mathrm{LVE}} \equiv \frac{G^{\prime}(\varphi)}{G^{\prime}(\varphi=0)}-1=2.5 \varphi+\delta \frac{G_{\mathrm{f}}^{\prime}}{G_{\mathrm{m}}^{\prime}} \frac{\varphi^{3}}{r}
$$

The subscripts $f$ and $m$ refer to the filler and matrix material, respectively, and $r$ is the effective radius of the fillers. $\delta$ is a dimensional coefficient that is close to $25 \mathrm{~nm}$ for both filled rubbers and the model system. The full data set is shown in Appendix A. We also show data on silica-filled rubbers from Baeza, et al. (2012) and Mujtaba, et al. (2014) in Appendix A; the modulus of the fillers is not given for these data, but if we assume that it is the same as that of the fillers used by MermetGuyennet et al. (2016), namely $10^{9} \mathrm{~Pa}$, then these data follow the same correlation with the same value of $\delta$. The $2.5 \varphi$ term 
in Eq. (1) is the usual "hydrodynamic" contribution. The second size-dependent term dominates outside a small linear regime, and additional terms that depend only on $\varphi$ but not on particle size do not contribute.

We examine here the nonlinear shear properties of the filled crosslinked systems up to strains of 0.6 , and for one filled rubber, we examine the extensional stress up to a Hencky strain of 0.47 . We find that the reinforcement given by Eq. 1 applies to the nonlinear properties as well, meaning that a good estimate of the full rheological response at modest but finite strains can be obtained solely from knowledge of the matrix properties and the size and modulus of the filler.

\section{Materials and methods}

\section{Filled rubbers}

We use three different types of filled rubbers. Each polymer matrix is made of styrene-butadiene rubber (SBR afterwards) provided by Michelin ${ }^{\circledR}\left(\rho=1.4 \times 10^{5} \mathrm{~g} / \mathrm{mol}\right.$ vulcanized $)$. The first (oiled) contains $30 \mathrm{phr}$ (parts per hundred rubber) of oil added to the SBR; the molecular weight of the oil is about 1000 times smaller than that of the SBR polymer. The addition of oil is a common practice in the rubber industry to improve the dispersion of the fillers. The second (non-functionalized) contains only SBR, and in the last (functionalized) $98 \%$ of the polymer chains carry a silanol-end group. The addition of the silanol-end group is to improve the compatibility of the polar silica fillers with the nonpolar rubber matrix. Each of these rubbers is filled with nanoparticles of precipitated silica (Zeosil 1165 MP, Rhodia ${ }^{\circledR}$ ). More information about the compounding and processing of the filled rubbers can be found (Baeza et al. 2012). Several examples of the importance of the storage modulus over the loss modulus can be found in (Mermet-Guyennet et al. 2015), with a ratio $\mathrm{G}^{\prime \prime} / \mathrm{G}^{\prime}$ about 0.1 . The modulus of the matrix and fillers can be found in Table 1 .

\section{Model system: filled polymer gel}

As a model system, a polymer gel is made by crosslinking polyvinyl alcohol (PVA) provided as a powder by Acros Organics (hydrolyzed at $99-100 \%$ and a molecular weight of $86,000 \mathrm{~g} / \mathrm{mol}$ ). The powder is dissolved (4\% wt.) in distilled water by mixing with a stirring bar at a temperature of $95^{\circ} \mathrm{C}$ for $3 \mathrm{~h}$. The crosslinker, borate, is obtained by dissolving sodium tetraborate (provided by Sigma Aldrich) in distilled water with a mass fraction of $8 \%$ wt. using the same mixing procedure as with PVA. The gel is finally formed by mixing $2 \mathrm{~g}$ of borate solution with $8 \mathrm{~g}$ of PVA solution. This model system is filled with non-surface treated polystyrene beads (Microbeads, Dynoseed (®) ranging from 20 to $250 \mu \mathrm{m}$ in radius and with a small polydispersity. It is important to notice that the particle size is much higher that the network formed by the gel. The modulus of the matrix and fillers can be found in Table 1 .

\section{Shear measurements}

Finite amplitude oscillatory measurements were performed using an Anton Paar Physica MCR 300 rheometer mounted with a plate/plate geometry at a frequency of $1 \mathrm{~Hz}$, which is within the range where the linear viscoelastic properties of the rubber and gel are insensitive to frequency (Mermet-Guyennet et al. 2015). For filled rubbers the diameter of the plates was $5 \mathrm{~mm}$ and the samples were disks with a thickness about $2.5 \mathrm{~mm}$, attached to the plates with a Loctite glue to avoid wall slip (Montes et al. 2003). The geometry was the same for experiments with the PVA gel, but we used a diameter of $25 \mathrm{~mm}$ and both surfaces were roughened to avoid wall slip. The shear stress is recorded at the maximum strain. The normal stress difference $N_{1}-N_{2}$ is obtained from the average normal force $\bar{F}_{N}$ over the cycle, which is the value reported by the rheometer software. All normal stresses are found to be quadratic over the entire strain range, in which case it readily follows that $N_{1}-N_{2}$ evaluated at the maximum strain is given by

$N_{1}-N_{2}=\frac{8 \bar{F}_{N}}{\pi r^{2}}$

\section{Tensile measurements}

Tensile measurements on the filled oiled rubber were performed using a Zwick Roell BZ2.5/TS1S tensile test
Table 1 Summary of the shear modulus of the polymer matrix and the fillers used in this article

\begin{tabular}{lll}
\hline System & Shear modulus of the matrix & $\begin{array}{l}\text { Shear modulus of the fillers } \\
\text { (approximation) }\end{array}$ \\
\hline Oiled & $5.10^{5} \mathrm{~Pa}$ & $10^{10} \mathrm{~Pa}$ \\
Non-functionalized & $1.10^{6} \mathrm{~Pa}$ & $10^{10} \mathrm{~Pa}$ \\
Functionnalized & $8.10^{5} \mathrm{~Pa}$ & $10^{10} \mathrm{~Pa}$ \\
Filled polymer gel & $10^{3} \mathrm{~Pa}$ & $10^{10} \mathrm{~Pa}$ \\
\hline
\end{tabular}


machine. The samples were strips with a thickness of $2.5 \mathrm{~mm}$ and a width of $1 \mathrm{~cm}$, clamped on both sides to be stretched. During these experiments the elongation rate was kept constant at $10^{-4} / \mathrm{min}$ and the samples were stretched from $\varepsilon=1$ until $\varepsilon=1.6$, where $\varepsilon$ is the engineering strain (the corresponding Hencky strain range is 0 to 0.47 ). We were unable to perform tensile experiments on the gel.

\section{Aggregate size measurements}

The silica particles exist as aggregates. Aggregate sizes were determined using SAXS experiments on the Dutch/ Belgian beam line (ID 26) at the ESRF (Grenoble, France), using a wave length of $1 \AA(12.4 \mathrm{keV})$ and a sample-to-detector distance of $7 \mathrm{~m}$. Additional information may be found in Appendix B.

\section{Filled rubbers: shear and normal stresses}

The shear stress of the oiled rubber at selected filler volume fractions of $0,0.11$, and 0.21 is shown as a function of strain in Fig. 1a. The stress is linear at small strains, after which there is strain softening for the filled rubbers (the Payne effect). The shear modulus is obtained from the fit to the small strain data, as shown in the insert. The same behavior is seen for the other two filled rubbers. Shear moduli obtained by fitting the linear regimes for the three filled rubbers are shown as functions of filler volume fraction in Fig. 1b. The normal stress difference $N_{1}-N_{2}$ of the oiled rubber at filler volume fractions of $0,0.11$, and 0.21 is shown as a function of strain squared in Fig. 1c; the normal stress remains quadratic over the entire strain range studied and, unlike the shear stress, shows little or no strain softening. The coefficient of the quadratic, denoted $B_{N}$, is shown in as a function of volume fraction in Fig. 1d for the three filled rubbers.

\section{Filled rubbers: tensile stresses}

The engineering tensile stress $\sigma_{z z}=F / A_{0}$ of the oiled rubber at filler volume fractions of $0,0.11$, and 0.21 is shown as a function of the engineering strain in Fig. 2. $F$ is the force exerted by the tensile test machine and $\mathrm{A}_{0}$ the initial cross-section. The engineering strain $\varepsilon$ is defined as the change in length divided by the initial length and is related to the Hencky strain $\varepsilon_{\mathrm{H}}$ through the relation $\varepsilon_{\mathrm{H}}=\ln (1+\varepsilon)$. The magnitude of the tensile stress increases with volume fraction at every strain, as expected.

\section{Model system: shear and normal stresses}

We now turn to the crosslinked polyvinyl alcohol gel reinforced with micron-scale spherical polystyrene beads of different sizes. The model system, despite its very simple chemical composition and microstructure, qualitatively
Fig. 1 a Stress versus strain for oiled-filled rubber at zero, intermediate, and high volume fractions $\varphi$. The inset shows a zoom on the linear regime (i.e., low strains) and the fit to obtain the shear modulus versus volume fraction shown in (b) for each filled rubber at each volume fraction. $\mathbf{c} \mathrm{N}_{1}-\mathrm{N}_{2}$ versus the square of the strain for the same samples as in (a). The lines are fits from which we obtain the prefactors $B_{N}$ plotted in (d)
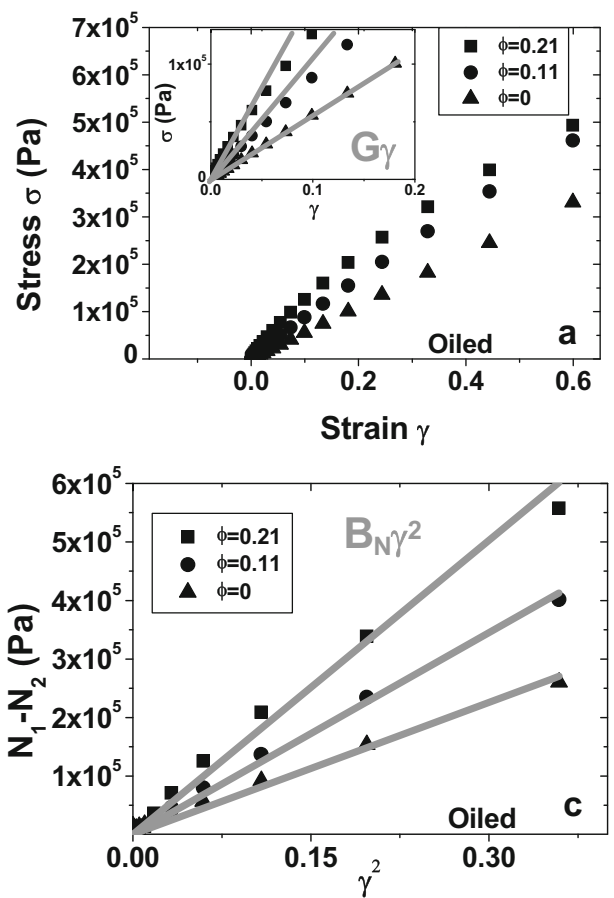

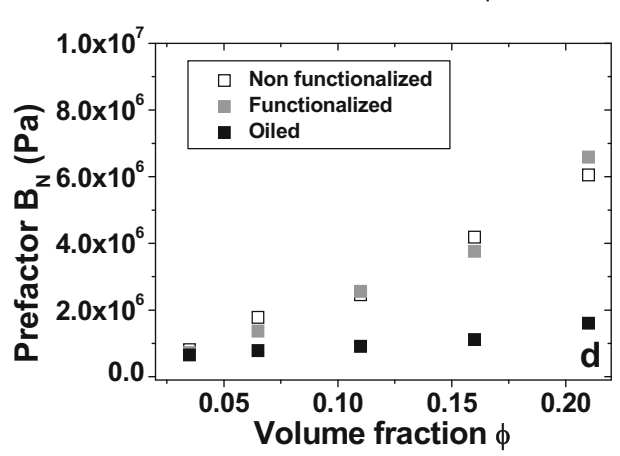




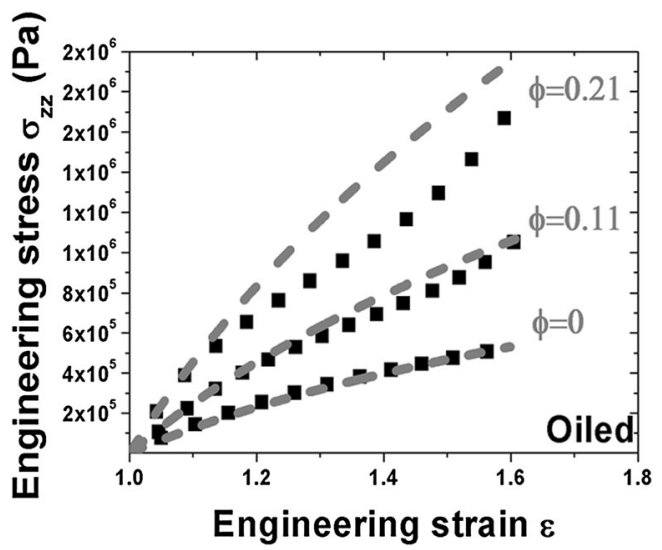

Fig. 2 Engineering stress obtained from tensile testing for the oiled-filled rubber at zero, intermediate, and high volume fraction. The dashed lines are Eq. (7)

exhibits the same mechanical behavior as the filled rubbers. The dilute regime in this system is wider (observable) than for filled rubbers and allows us to understand step by step the normal stresses in our composite material.

Figure $3 \mathrm{a}, \mathrm{c}$ shows the shear and normal stress at different particle volume fractions for the model system. As with the unfilled rubber, the PVA gel exhibits a linear stress-strain relation and a positive normal stress that varies quadratically with strain. With the addition of fillers the shear and normal stresses increase with volume fraction at fixed strain; the modulus becomes nonlinear but the normal stress difference remains quadratic, as with the rubbers.

\section{A universal rescaling}

The storage moduli of the rubbers and the model gel are much larger than the loss moduli, with $\mathrm{G}^{\prime} / \mathrm{G}^{\prime \prime}$ typically of order 10 (Mermet-Guyennet et al. 2015), so the low frequency value of $\mathrm{G}^{\prime}$ is expected to equal the shear modulus $\mathrm{G}$ at small strains, and this is indeed the case for all systems studied here. Hence, the reinforcement $R$ is identical to the linear viscoelastic reinforcement $R_{\mathrm{LVE}}$ given by Eq. (1), as shown in Fig. 4a, $\mathrm{b}$, and we have

$R=R_{\mathrm{LVE}}=\mathrm{G}(\phi) / \mathrm{G}(\phi=0)-1=2.5 \varphi+\delta \frac{G_{\mathrm{f}}}{G_{\mathrm{m}}} \frac{\varphi^{3}}{r}$

with $\delta=25 \mathrm{~nm}$ for both systems.

We now turn to the normal stresses. We introduce by analogy the normal reinforcement $R_{\mathrm{N}}$,

$R_{N}=B_{N}(\phi) / B_{N}(\phi=0)-1$

As is evident from the insert in Fig. 3d, the normal reinforcement follows the classical result $R_{N}=2.5 \phi$ for the model system in the dilute region, followed by a nonlinear dependence at higher volume fractions where multiple particle effects become important. We show the normal reinforcement plotted as a function the reinforcement in Fig. 5 for all systems studied, both the rubbers and the model gel, and it is clear that $R_{N}=R=R_{\mathrm{LVE}}$. The normal reinforcement in fact follows the universal scaling law over the entire strain range studied, even
Fig. 3 a Stress versus strain for the model system with an average particle radius of $125 \mu \mathrm{m}$ at zero, intermediate, and high volume fraction. The inset shows a zoom on the linear regime (i.e., low strains), and the fit to obtain the shear modulus versus volume fraction shown in (b) for each particle radius. c $\mathrm{N}_{1}-\mathrm{N}_{2}$ versus the square of the strain for the same samples as in (a). The lines are fits from which we obtain the prefactors $B_{N}$ plotted in (d)
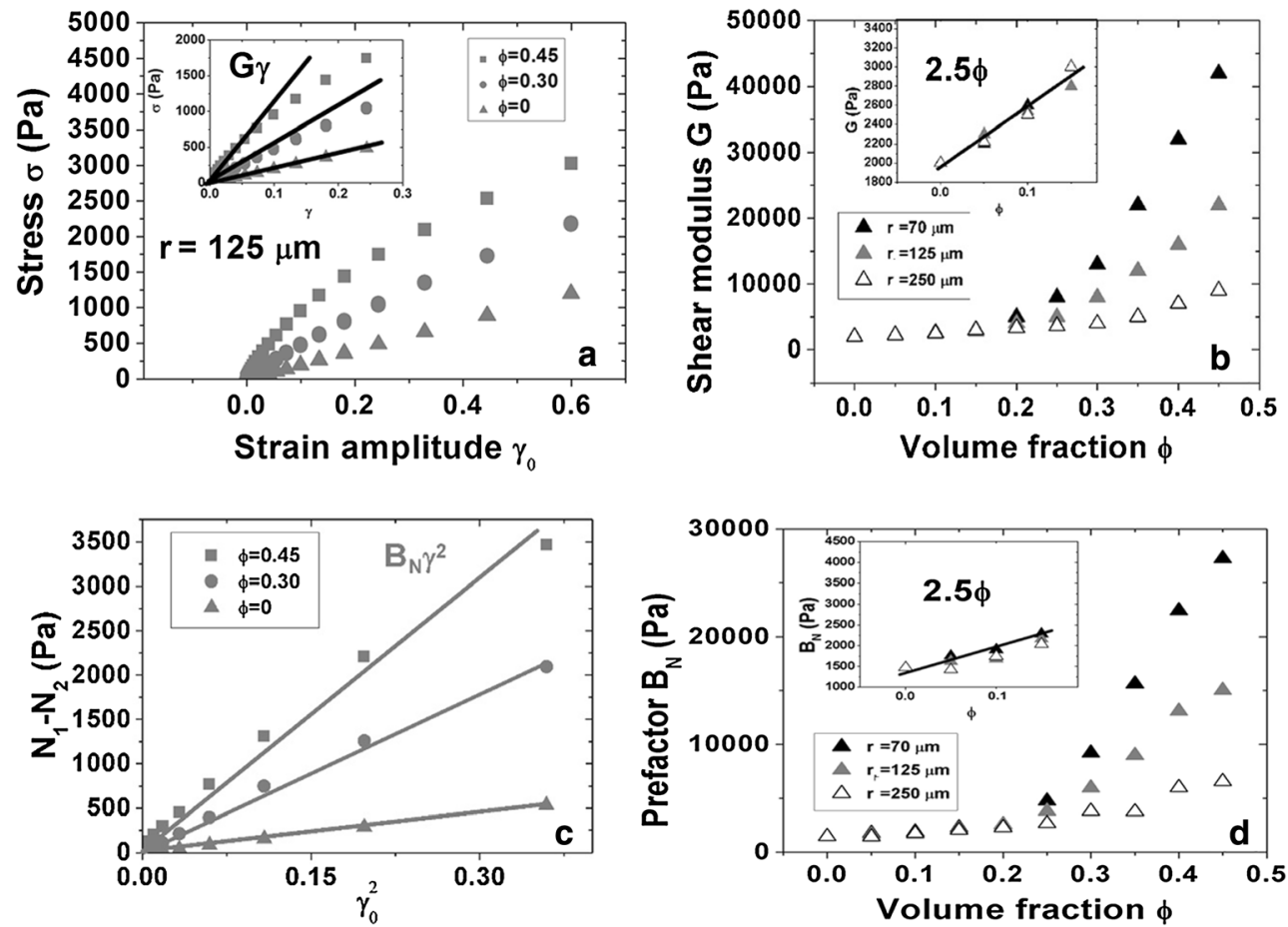
Fig. 4 Relative increase of the modulus due to the addition of filler particles versus the interaction term of the previously introduced rescaling for the silicafilled rubbers (a) and for the model system of the PVA gel filled with polystyrene particles (b). The "hydrodynamic" contribution has been subtracted from $R$ to isolate the interaction term. The value of delta is the same for both graphs

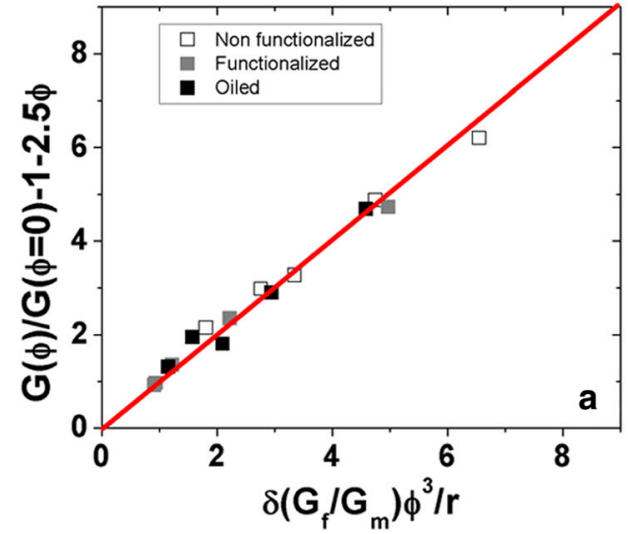

in the regime where the shear modulus exhibits the Payne effect and is strain softening.

\section{Mooney-RIVLIN model}

The deformation of rubbers up to strains of order unity is frequently characterized by the Mooney-Rivlin model (Gent and Thomas 1958, Mooney 1940, Rivlin 1948). The total strain energy density for incompressible Mooney-Rivlin materials is given by

$W=C_{1}\left(I_{1}-3\right)+C_{2}\left(I_{2}-3\right)$

with $C_{1}$ and $C_{2}$ as the two Mooney-Rivlin material constants, and $\mathrm{I}_{1}$ and $\mathrm{I}_{2}$ as the first and second invariants, respectively, of the left Cauchy-Green tensor. The shear modulus G and normal stress coefficient $B_{N}$ measured in torsional shear are related to the two Mooney-Rivlin coefficients by

$G=2\left(C_{1}+C_{2}\right)$

and

$B_{N}=2 C_{1}+4 C_{2}$.

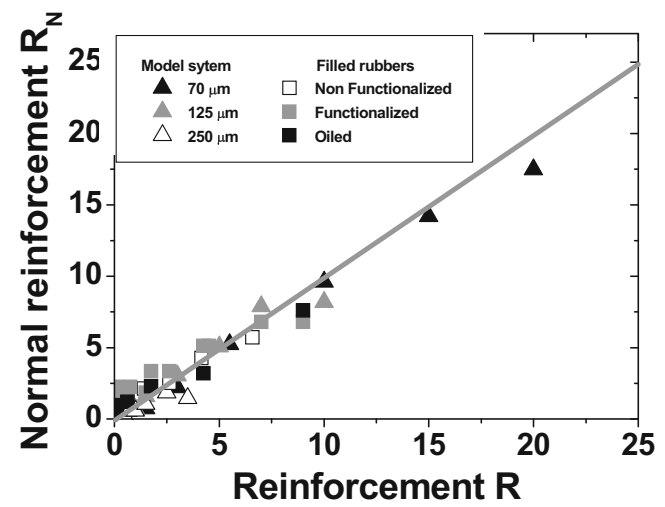

Fig. 5 Normal reinforcement plotted versus reinforcement for the filled rubbers and model systems. The line has unit slope and passes through the origin
Since $G$ and $B_{N}$ follow the scaling in Eq. (3), it is obvious from the linearity of Eq. (6) that the Money-Rivlin coefficients must follow the same scaling. The Mooney-Rivlin coefficients of the oiled-rubber samples in Fig. 1a, c are recorded in Table 2.

The engineering tensile stress for a Mooney-Rivlin material as a function of the engineering strain is given by

$\sigma_{z z}=2\left(\varepsilon-\varepsilon^{-2}\right)\left(C_{1}+C_{2} \varepsilon^{-1}\right)$.

The prediction of Eq. (7) for the oiled-rubber samples using the Mooney-Rivlin coefficients in Table 1 is plotted in Fig. 2, together with the experimental data. The agreement with the Mooney-Rivlin prediction for the unfilled oiled rubber is good over the entire range of engineering strain. Agreement for the filled rubber is good at low strains, but the theoretical curves for the filled polymers based on the shear data capture the extensional stresses of the filled rubber only up to engineering strains of about 1.1 and 1.2 for the 0.11 and 0.21 volume fractions, respectively (i.e., Hencky strains of about 0.1 and 0.18 , respectively), after which they overpredict the tensile stresses and do not capture the inflection and subsequent extensional strain hardening. The fact that the Mooney-Rivlin coefficients follow the $\phi^{3} / \mathrm{r}$ scaling means that the stress response to any small but finite deformation for a filled system can be determined simply from knowledge of the rheological properties of the matrix and the modulus of the filler.

Table 2 Calculation of the Mooney Rivlin coefficients $\mathrm{C}_{1}$ and $\mathrm{C}_{2}$ from the experimental values of $G$ and $B_{N}$ for oiled rubber at three volume fractions $\varphi$

\begin{tabular}{lllll}
\hline & $G(\mathrm{~Pa})$ & $B_{N}(\mathrm{~Pa})$ & $C_{1}(\mathrm{~Pa})$ & $C_{2}(\mathrm{~Pa})$ \\
\hline$\varphi=0$ & $5.5 \times 10^{5}$ & $0.7 \times 10^{6}$ & $2 \times 10^{5}$ & $0.75 \times 10^{5}$ \\
$\varphi=0.11$ & $8.9 \times 10^{5}$ & $1.1 \times 10^{6}$ & $3.4 \times 10^{5}$ & $1.05 \times 10^{5}$ \\
$\varphi=0.21$ & $16 \times 10^{5}$ & $1.9 \times 10^{6}$ & $6.5 \times 10^{5}$ & $1.5 \times 10^{5}$ \\
\hline
\end{tabular}




\section{Discussion}

This powerful $\phi^{3} / \mathrm{r}$ scaling for the rheology of filled systems at small but finite deformations is not predicted by conventional homogenization analyses, which are scale-free. A modulus scaling of roughly $\phi^{3}$ has been observed for percolated networks (Forsman, et al. 1987), but the systems considered here are below the usual percolation threshold, and percolation of the particulates is not observed in micrographs (Mermet-Guyennet et al. 2016). $\phi / r$ is the surface area/unit volume, and its presence multiplied by the modulus ratio indicates a force that scales with the number of polymer chains in the neighborhood of the particle. $\phi^{2}$ is expected as the lowest order contribution for multiple particle interactions, hence the $\phi^{3} / r$ dependence is reasonable as the first correction to the "hydrodynamic" $2.5 \phi$ term. This picture is conceptually similar to the mechanism for reinforcement proposed by Akcora and coworkers (Akcora, et al. 2009, Moll, et al. 2011, Zhao et al. 2015) for grafted nanoparticles in uncrosslinked polymer melts. Notably, Zhao and coworkers (Zhao, Ge, Senses, Akcora, Jestin, and Kumar 2015) have observed a $\phi^{3}$ scaling for the storage modulus of silica nanoparticles in poly(2-vinylpyridine), together with a surprisingly small percolation threshold. Davris et al. (Davris, et al. 2016) have shown through molecular simulations on thin films and particulate systems that at high enough volume fraction the mechanism leading to a strong reinforcement is the formation of a filler network rather than a confinement of the polymer chains between two surfaces, but it is not apparent that the simulations are directly relevant to the observations reported here. A true mechanistic explanation of this remarkable behavior that is derived from first principles is still needed. In particular, it is not obvious why the characteristic length scale should be of order $25 \mathrm{~nm}$ for such different crosslinked polymer systems and particle sizes. It is clear that Eq. (3) cannot apply as $\phi$ approaches maximum packing, but that limit is not relevant in filled rubber applications.

Acknowledgements This work is part of the research program "Understanding the visco-elasticity of elastomer based nanocomposites" (11VEC01) of the Stichting voor Fundamenteel Onderzoek der Materie (FOM), which is financially supported by the Nederlandse Organisatie voor Wetenschappelijk Onderzoek (NWO). MMD acknowledges a stimulating conversation with Sanat Kumar
Appendix A

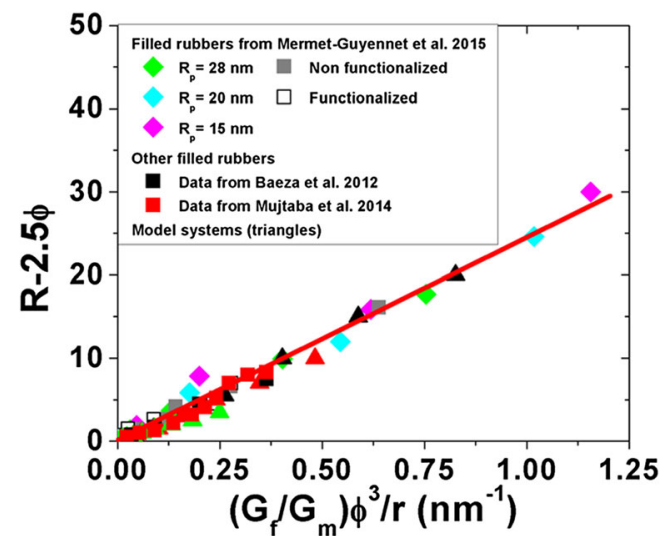

Fig. 6 Reinforcement data from Mermet-Guyennet et al. 2015. Two of the filled rubbers are considered in this article, while the three filled rubbers with different particle radii $(R \mathrm{p})$ are nitrile-butadiene rubber with silica particles. The model system includes the same filled PVA formulation that considered in the present paper. In addition, we compare our previous data to those from Baeza et al. 2012 and Mujtaba et al. 2014. The red line shows the fit to determine $\delta$, which has a value of $25 \mathrm{~nm}$

\section{Appendix B}

The SAXS experiments were carried out in the Dutch/ Belgian beam line (ID 26) at the ESRF (Grenoble, France). They were performed using a wave length of $1 \mathrm{~A}(12.4 \mathrm{keV})$ and a sample-to-detector distance of $7 \mathrm{~m}$. Samples were cut into 8 -mm-diameter disks with a thickness about $2 \mathrm{~mm}$. The shape of the scattering patterns for filled rubbers is quite complicated, but if we assume that the aggregates and the primary particles are spherical and monodisperses at high $q$ a first break in slope corresponds to the size of the primary particle $\left(q_{\mathrm{Si}}=\pi / r_{\mathrm{Si}}\right.$ with $r_{\mathrm{Si}}$ the primary particle radius) and at medium $q$ a second break in slope corresponds to the size of the aggregates $\left(q_{\mathrm{agg}}=\pi / r_{\mathrm{agg}}\right.$ with $r_{\mathrm{agg}}$ the aggregate radius). A more complete description of the rich microstructure of the aggregates in filled rubbers and the way to interpret the SAXS spectra from this system can be found in (Baeza, Genix, Degrandcourt, Petitjean, Gummel, Couty and Oberdisse 2012). 
Fig. 7 SAXS spectra for nonfunctionalized filled rubber (a). The inset shows the same spectra using a Kratky representation. The maximum on the inset correspond to the aggregate radius. b Aggregate radius versus volume fraction for the different filled rubbers considered in this article. The volume fraction dependence is not surprising and has been discussed elsewhere (Bumm, et al. 2014)

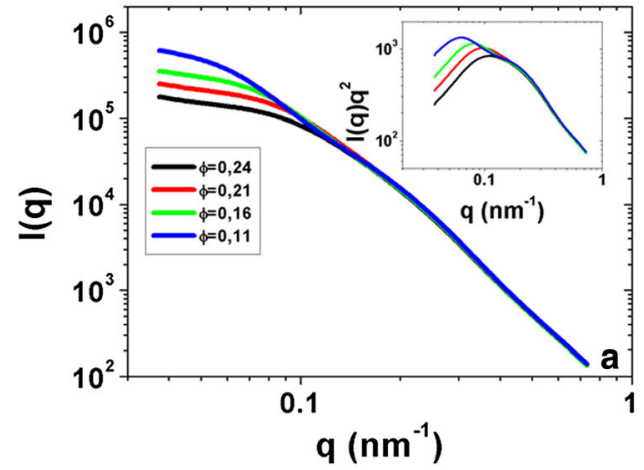

Open Access This article is distributed under the terms of the Creative Commons Attribution 4.0 International License (http:// creativecommons.org/licenses/by/4.0/), which permits unrestricted use, distribution, and reproduction in any medium, provided you give appropriate credit to the original author(s) and the source, provide a link to the Creative Commons license, and indicate if changes were made.

\section{References}

Akcora P, Kumar SK, Moll J, Lewis S, Schadler LS, Li Y, Benicewicz BC, Sandy A, Narayanan S, Ilavsky J (2009) "Gel-like" mechanical reinforcement in polymer nanocomposite melts. Macromolecules 43:1003-1010

Baeza GP, Genix A-C, Degrandcourt C, Petitjean L, Gummel J, Couty M, Oberdisse J (2012) Multiscale filler structure in simplified industrial nanocomposite silica/SBR systems studied by SAXS and TEM. Macromolecules 46:317-329. doi:10.1021/ma302248p

Bumm SH, White JL, Isayev AI (2014) Breakup of silica agglomerates in corotating twin-screw extruder: modeling and experiment. Journal of Elastomers and Plastics 46:527-552. doi:10.1177/ 0095244313476508

Chen Q, Gong S, Moll J, Zhao D, Kumar SK, Colby RH (2015) Mechanical reinforcement of polymer nanocomposites from percolation of a nanoparticle network. ACS Macro Lett:398-402. doi:10. 1021/acsmacrolett.5b00002

Davris T, Mermet-Guyennet MR, Bonn D, Lyulin AV (2016) Filler size effects on reinforcement in elastomer-based nanocomposites: experimental and simulational insights into physical mechanisms. Macromolecules 49:7077-7087

Forsman J, Harrison JP, Rutenberg A (1987) Elasticity of a percolation system: silica smoke. Can J Phys 65:767-771. doi:10.1139/p87-112

Fröhlich J, Niedermeier W, Luginsland HD (2005) The effect of fillerfiller and filler-elastomer interaction on rubber reinforcement. Compos A: Appl Sci Manuf 36:449-460. doi:10.1016/j. compositesa.2004.10.004

Gent AN, Thomas AG (1958) Forms for the stored (strain) energy function for vulcanized rubber. J Polym Sci 28:625-628. doi:10.1002/ pol.1958.1202811814

Guth E (1945) Theory of filler reinforcement. Rubber Chem Technol 18: 596-604. doi:10.5254/1.3546754
Mermet-Guyennet M, Gianfelice de Castro J, Varol HS, Habibi M, Hosseinkhani B, Martzel N, Sprik R, Denn MM, Zaccone A, Parekh SH, Bonn D (2016) Size-dependent reinforcement of composite rubbers. Polymer 73:170-173. doi:10.1016/j.polymer.2015. 07.041

Mermet-Guyennet M, Gianfelice de Castro J, Habibi M, Martzel N, Denn M, Bonn D (2015) LAOS: the strain softening/strain hardening paradox. J Rheol 59:21-32

Moll JF, Akcora P, Rungta A, Gong S, Colby RH, Benicewicz BC, Kumar SK (2011) Mechanical reinforcement in polymer melts filled with polymer grafted nanoparticles. Macromolecules 44:7473-7477

Montes H, Lequeux F, Berriot J (2003) Influence of the glass transition temperature gradient on the nonlinear viscoelastic behavior in reinforced elastomers. Macromolecules 36:8107-8118. doi:10.1021/ $\mathrm{ma} 0344590$

Mooney M (1940) A theory of large elastic deformation. J Appl Phys 11: 582-592. doi:10.1063/1.1712836

Mujtaba A, Keller M, Ilisch S, Radusch H-J, Beiner M, Thurn-Albrecht T, Saalwachter K (2014) Detection of surface-immobilized components and their role in viscoelastic reinforcement of rubber-silica nanocomposites. ACS Macro Lett 3:481-485

Payne AR (1962) The dynamic properties of carbon black-loaded natural rubber vulcanizates. Part I Journal of Applied Polymer Science 6: 57-63. doi:10.1002/app.1962.070061906

Raos G (2003) Application of the Christensen-Lo model to the reinforcement of elastomers by fractal fillers. Macromolecular Theory and Simulations 12:17-23. doi:10.1002/mats.200390002

Rattanasom N, Saowapark T, Deeprasertkul C (2007) Reinforcement of natural rubber with silica/carbon black hybrid filler. Polym Test 26: 369-377

Rivlin RS (1948) Large elastic deformations of isotropic materials. IV. Further developments of the general theory. Philosophical Transactions of the Royal Society of London Series A, Mathematical and Physical Sciences 241:379-397. doi:10.1098/ rsta. 1948.0024

Sadd MH (2009) Elasticity: theory, applications, and numerics. Academic Press

Smallwood HM (1944) Limiting law of the reinforcement of rubber. J Appl Phys 15:758-766. doi:10.1063/1.1707385

Treloar LRG (1975) The physics of rubber elasticity. Oxford University Press

Zhao D, Ge S, Senses E, Akcora P, Jestin J, Kumar SK (2015) Role of filler shape and connectivity on the viscoelastic behavior in polymer nanocomposites. Macromolecules 48:5433-5438 